\title{
SUPERIMPOSED ANATOMICAL STRUCTURES IN AUGMENTED REALITY
}

Vladimir Ivanov

Herzen State Pedagogical University of Russia,

Saint-Petersburg, (Russia).

E-mail:voliva@rambler.ru ORCID: https://orcid.org/0000-0001-8194-2718

Nikolay Kalakutskiy

Pavlov First State Medical University of St. Petersburg,

Saint-Petersburg, (Russia).

E-mail: kalakutsky@yandex.ru ORCID: https://orcid.org/0000-0002-6851-5073

Alexander Klygach

Herzen State Pedagogical University of Russia,

Saint-Petersburg, (Russia).

E-mail: voolf00@yandex.ru ORCID: https://orcid.org/0000-0002-2984-0201

Sergey Strelkov

Herzen State Pedagogical University of Russia,

Saint-Petersburg, (Russia).

E-mail: sergin3d2d@gmail.com ORCID: https://orcid.org/0000-0002-4830-5407

\section{Citación sugerida Suggested citation}

Ivanov, V., Kalakutskiy, N., Klygach, A., y Strelkov, S. (2020). Superimposed anatomical structures in augmented reality. 3C Tecnología. Glosas de innovación aplicadas a la pyme. Edición Especial, Noviembre 2020, 21-31. https://doi.org/10.17993/3ctecno.2020.specialissue6.21-31 


\section{ABSTRACT}

The paper considers an approach to visualization of anatomical structures using augmented reality, which allows for more accurate planning of operations and reduces the degree of surgical intervention.

To achieve this goal, a technology was developed using a frame with a volumetric optical marker, which allows you to correlate a virtual 3D model with the patient. As a result, it was possible to achieve an accurate positioning of the 3D model and obtain a reliable visualization of the location of internal anatomical structures. At the same time, the parallel application of spatial mapping technology allows you to fix the 3D model at a certain point in space and, in case of loss of marker tracking, save the correct location of the 3D model, taking into account that the patient is in a stationary state.

This approach can be effectively applied in surgery, if it is possible to fix the skeleton relative to any bone structure. Options for surgical intervention can be different, starting with the installation of prostheses, ending with the removal of cancerous tumors. The article discusses a project to develop a technology for using augmented reality in planning and conducting maxillofacial surgery. The capabilities of the technology, as well as the prospects for its use, are highlighted.

\section{KEYWORDS}

Medicine, Surgery, Maxillofacial surgery, Augmented reality, Markers, 3D graphics, Visualization. 


\section{INTRODUCTION}

Worldwide, the modern standard for jaw cancer surgery is the simultaneous reconstruction of a defect with a vascular graft. To reduce the risk of intra- and postoperative complications, improve the postoperative quality of life of patients and increase the accuracy of operations, much attention is paid to their careful preoperative planning (Hartman, Spauwen, \& Jansen, 2002). The complexity of planning reconstructive operations in oncology lies in the need to combine data from different studies to simultaneously visualize several body tissues (bones, blood vessels, nerves, soft tissues) in order to increase the objectivity of determining the boundaries of the resection, optimizing the choice of a vascularized graft and determining donor and recipient vessels. All the above tasks correspond to an offline "map" of the patient, used in the developed augmented reality program, which is compiled on the basis of CT or MRI data and can be supplemented by ultrasound, dopplerography, angiography, etc.

Augmented Reality (AR) is a combination of virtual reality and with the "offline map" of a patient and allows the user to interact in real time between various material and virtual objects, as if they exist in a single environment (Bartella et al., 2019). Using this feature, we can increase the productivity and quality of services in many areas, especially in head and neck surgery. The proposed solution will help the doctor easily see where the internal anatomical structures are located during the operation. We offer an advanced solution for tracking optical markers with spatial imaging based on patient CT data. The surgeon during the operation through glasses sees a $3 \mathrm{D}$ visualization of the received data, due to which he less looks at the monitor with the data of diagnostic tests and constantly monitors the state of the surgical wound, which reduces the risk of intraoperative complications, including bleeding, which leads to an increase in the accuracy of surgery, reducing its duration and reducing the risk of complications. Also, this project can be widely used for educational purposes.

Currently, many scientific papers on the use of augmented reality in various fields of medicine have been published: neurosurgery, cardiology, urology, plastic surgery, including dentistry and maxillofacial surgery (Ayoub \& Pulijala, 2019; Elmi-Terander et al., 2020; Kim, Kim, \& Kim, 2017; Kwon, Park, \& Han, 2018). However, most of them are descriptions 
of individual clinical cases or their series, which certainly does not have a very high class of evidence (Bosc et al., 2019). Available literature reviews also note the lack of clinical trial results for a more objective assessment of this method. Therefore, the goal of our work is to conduct a multicenter randomized clinical trial in the treatment of patients with jaw cancer, which shows their resection with simultaneous plastic surgery of the defect with a vascularized bone graft with microtechnology. A feature of basic research is their careful planning, strict adherence to the research protocol, good statistical analysis of the data obtained, as well as a unified scale for assessing the data obtained (calibrated investigator) and their analysis by an uninvolved specialist, to reduce the likelihood of bias on the part of the scientist when interpreting the data, compliance All of the above conditions will allow you to perform a high-class evidence study, the results of which will be useful for the development of modern science.

On the basis of the Department of Maxillofacial and Reconstructive Surgery of the First St. Petersburg Medical University named after Acad. I.P. Pavlova reconstructive plastic surgery to eliminate jaw defects due to malignant and benign neoplasms, post-traumatic defects and deformations of various genesis with the help of vascularized bone and softtissue flaps with microsurgical technique has been carried out for more than 30 years by a team led by professor N.V. Kalakutsky During this time, vast clinical experience has been accumulated in the treatment of this group of patients, which will facilitate the introduction of new technologies and improve the quality of the study.

According to the example of the following clinical case of using augmented reality technology in dentistry, within the framework of the planned study, it is supposed to be used when performing a jaw resection for cancer with simultaneous plastic surgery of the defect with a bone graft with microtechnology.

\section{METHODOLOGY}

\subsection{MEDICAL CASE}

For the first case, we took a patient who has a small hollow cluster of cells that is grouped together - a cyst in the left nasal sinus (Figure 1), which was recommended for removal. It also has a complex and uneven shape. Therefore, to help the doctor better understand 
where he is during planning and work, we have developed a visual solution with the display of anatomical structures on top of what the doctor sees.

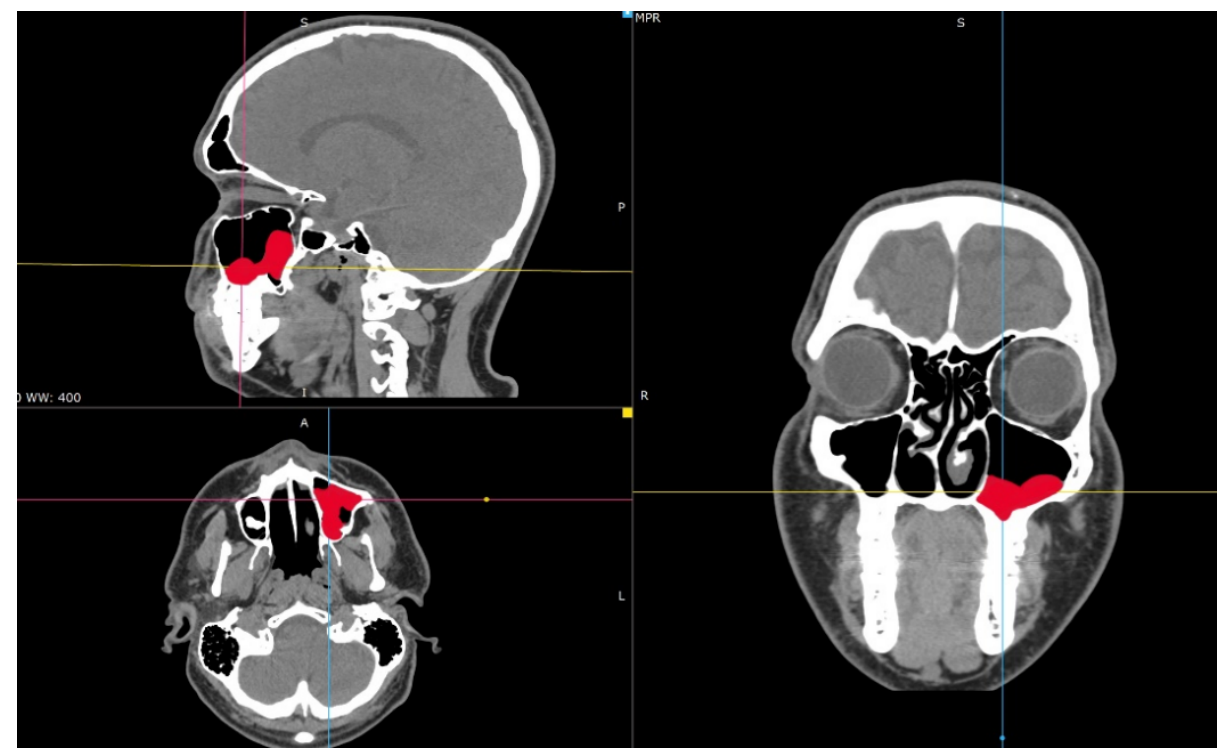

Figure 1. Cyst in left sinus marked in red.

\subsection{OVERLAY 3D MODELS OF THE SKULL IN THE REAL WORLD}

For the selected task, Augmented Reality is the optimal visual solution.

Augmented Reality (AR) presents information in the right context of the real world. To do this, the system must know where the user is and what he is looking at. Usually the user observes an object through a display that displays a camera image along with augmented reality. Thus, in practice, the system needs to determine the location and orientation of the camera relative to the object. In the case of Hololens glasses, it is almost the same. To do this, use a separate built-in camera, and as a display - two holographic projectors.

In AR technology, tracking markers are used for display on the display and in the camera AR codes that help align the $3 \mathrm{~d}$ model from the user's point of view. In our problem, when an object should be viewed from different sides, we used a special marker called cuboid, where on each side there is a unique AR code. The Hololens camera has a limited resolution of $720 \mathrm{p}$ and a low dynamic range compared to modern cameras. This causes problems 
with accurate tracking. To improve this, we have added the wrong organic structure, which provides additional tracking capabilities.

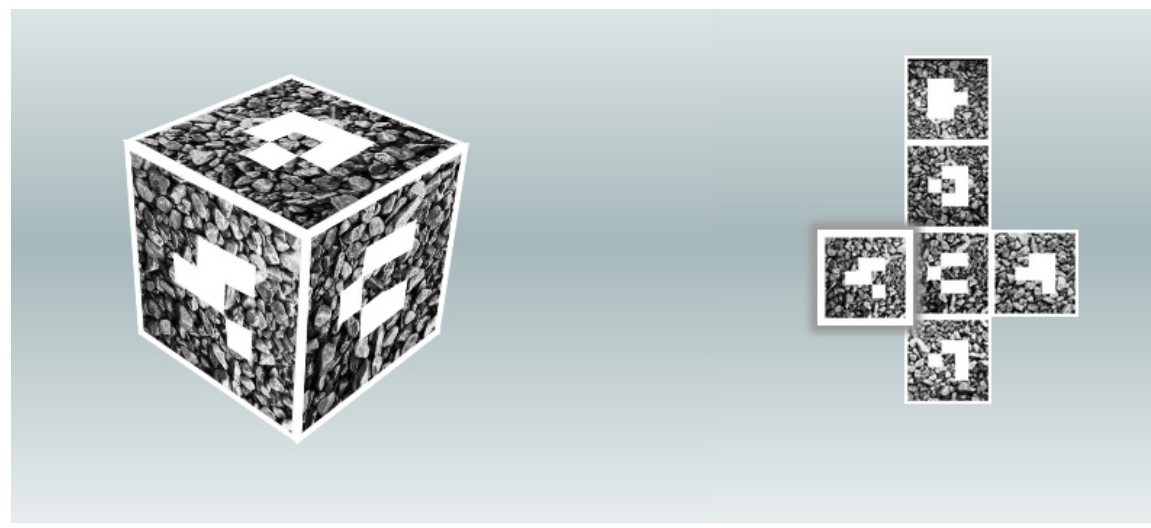

Figure 2. Type of marker.

In addition to the problem of aligning the object with the camera, our case is complicated by the fact that the marker must be fixed on the person's head, strictly in a certain position and with a certain orientation. For this task, we built a 3D model of the head from CT data using the Materialize Mimics software. Then we designated the areas where the marker will align and fix. Based on the selected areas, a three-dimensional wireframe model was built with a platform for attaching a marker. The frame was made in such a way that it could be easily put on and fixed in the correct position (Figure 3). Finally, it was printed on a 3D printer (Figure 4), and the marker was attached at a predetermined location.

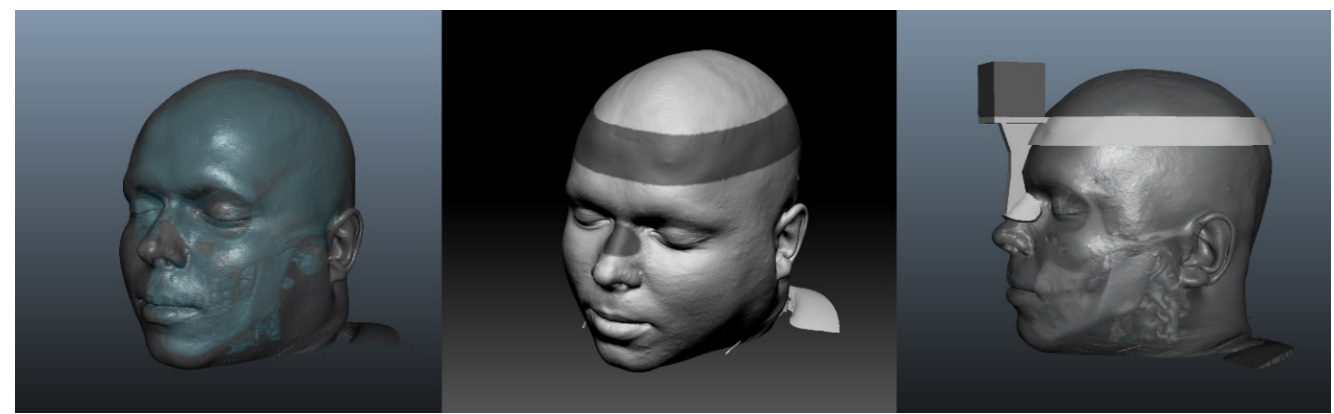

Figure 3. Left image - A constructed model of the face and skull based on CT data; in the middle are designated areas for fixing the frame, on the right is the model of the frame with a zone for the marker on top. 


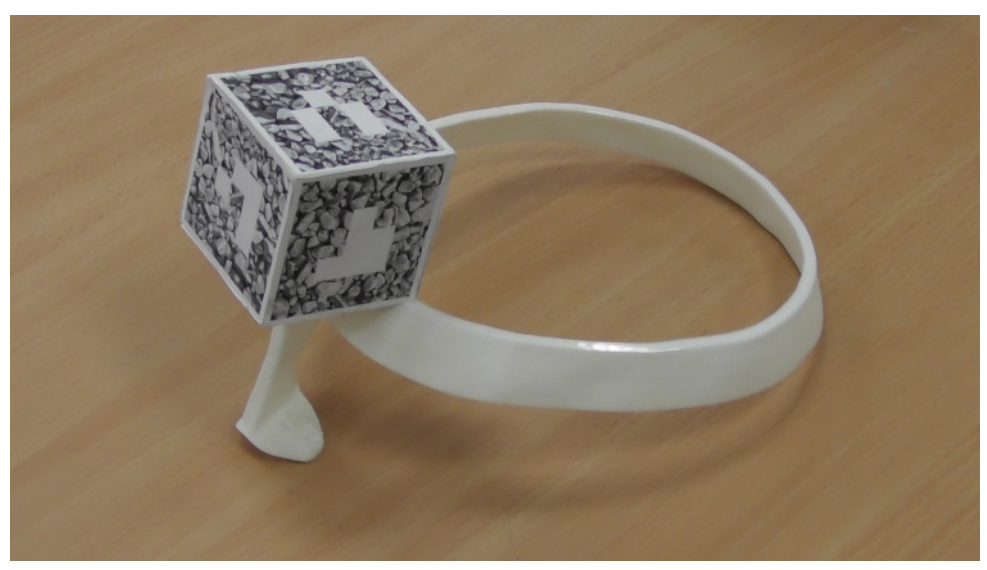

Figure 4. Printed frame.

After that, using the same CT dataset, we extracted a model of the patient's skull and cysts using Mimics. In the case of a cyst, it was isolated by CT by the doctor in layers, because such structures are clearly not readable on CT, and the program cannot automatically detect them. These two models were exported to Unity for further development under Hololens glasses (Figure 5).

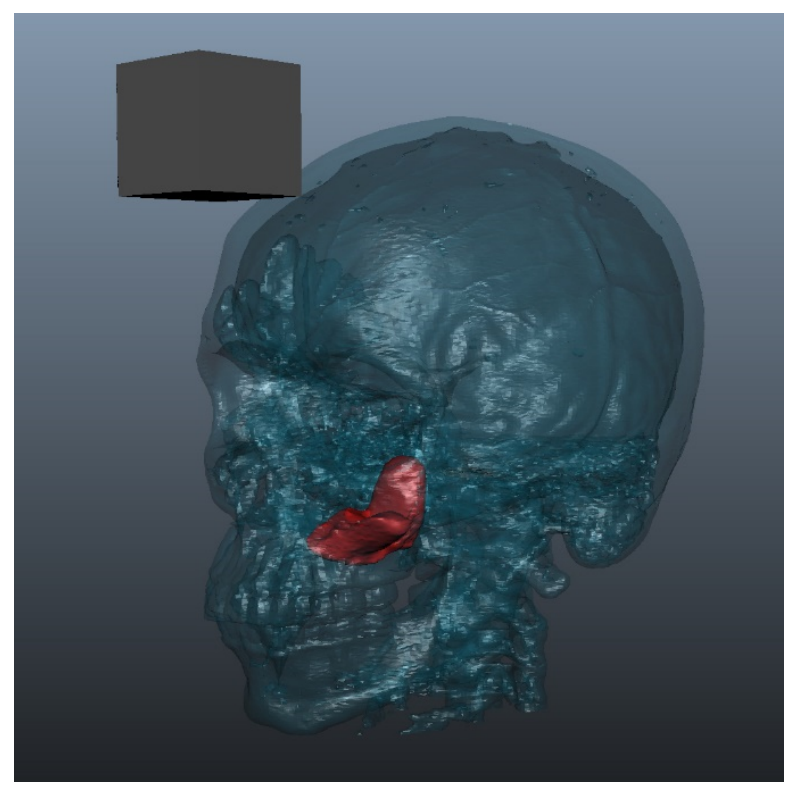

Figure 5. 3D model of a skull and cyst for augmented reality. The cube determines the size and position of the marker. 


\section{RESULTS}

\subsection{APPLICATION DEVELOPMENT FOR HOLOLENS}

We developed the application using the Unity environment, which has special tools for HoloLens. There are also additional modules for Unity that expand the capabilities. One of them is Vuforia, designed for augmented reality using marker tracking.

In addition, a simple user interface was added using a gesture that the glasses recognize - a click to hide the skull and a click and drag to change the opacity when viewing the hologram.

To improve tracking performance, we also used a feature called spatial mapping, which is available in HoloLens. Spatial mapping provides additional information about the position and orientation of glasses in the environment with high accuracy.

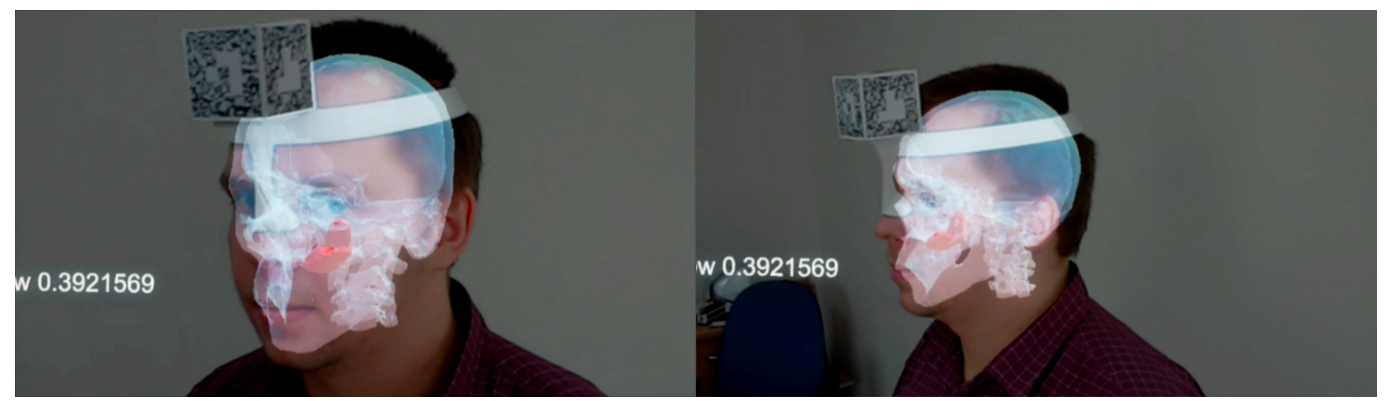

Figure 6. View of the skull and cyst through the HoloLens glasses.

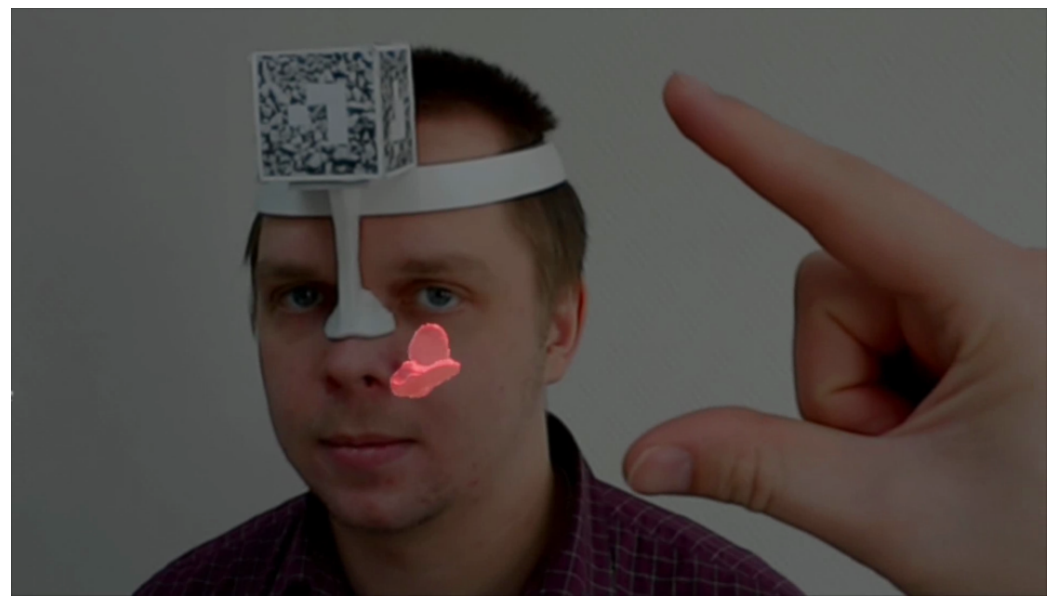

Figure 7. View of an isolated cyst through HoloLens glasses. 


\section{CONCLUSIONS}

The proposed approach using holographic representation of anatomical structures can be potentially used in surgery planning and during a procedure with some restrictions. Currently, marker based solutions on HoloLens devices have few limitations, such as low resolution cameras that are sensitive to lighting conditions and insufficient computing power of glasses. This causes a delay between 100-500 milliseconds and produces an error threshold in hologram positioning between $1-5 \mathrm{~mm}$. Such problems can be solved by upgrading hardware in the future glasses releases and possibly by switching to a geometry tracking solution, where markers can be represented by geometrical objects with any shape.

\section{REFERENCES}

Ayoub, A., \& Pulijala, Y. (2019). The application of virtual reality and augmented reality in Oral \& Maxillofacial Surgery. BMC Oral Health, 19(1), 238. https://doi. org/10.1186/s12903-019-0937-8

Bartella, A. K., Kamal, M., Scholl, I., Schiffer, S., Steegmann, J., Ketelsen, D., Hölzle, F, \& Lethaus, B. (2019). Virtual reality in preoperative imaging in maxillofacial surgery: implementation of "the next level"? British fournal of Oral and Maxillofacial Surgery, 57(7), 644-648. https://doi.org/10.1016/j.bjoms.2019.02.014

Bosc, R., Fitoussi, A., Hersant, B., Dao, T. H., \& Meningaud, J. P. (2019). Intraoperative augmented reality with heads-up displays in maxillofacial surgery: a systematic review of the literature and a classification of relevant technologies. Int f Oral Maxillofac Surg, 48(1), 132-139. https://doi.org/10.1016/j.ijom.2018.09.010

Elmi-Terander, A., Burström, G., Nachabé, R., Fagerlund, M., Ståhl, F., Charalampidis, A., Edström, E., \& Gerdhem, P. (2020). Augmented reality navigation with intraoperative $3 \mathrm{D}$ imaging vs fluoroscopy-assisted free-hand surgery for spine fixation surgery: a matched-control study comparing accuracy. Scientific Reports, 10(1), 707. https://doi.org/10.1038/s41598-020-57693-5 
Hartman, E. H., Spauwen, P. H., \& Jansen, J. A. (2002). Donor-site complications in vascularized bone flap surgery. Fournal of Investigative Surgery, 15(4), 185-197. https:// doi.org/10.1080/08941930290085967

Kim, Y., Kim, H., \& Kim, Y. O. (2017). Virtual Reality and Augmented Reality in Plastic Surgery: A Review. Archives of Plastic Surgery, 44(3), 179-187. https:/ / doi.org/10.5999/ aps.2017.44.3.179

Kwon, H. B., Park, Y. S., \& Han, J. S. (2018). Augmented reality in dentistry: a current perspective. Acta Odontologica Scandinavica, 76(7), 497-503. https://doi.org/10.1080/0 0016357.2018 .1441437 
3C Tecnología. Glosas de innovación aplicadas a la pyme. ISSN: 2254 - 4143 Edición Especial Special Issue Noviembre 2020 